\title{
Spin Dynamics and Low Energy Vibrations: Insights from Vanadyl- Based Potential Molecular Qubits
}

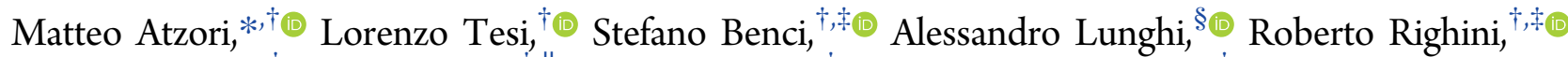 \\ Andrea Taschin, ${ }^{\ddagger}$ Renato Torre, ${ }^{\ddagger}, \|$ Lorenzo Sorace, ${ }^{\dagger}$ and Roberta Sessoli*, ${ }^{\dagger}$ (D) \\ †Dipartimento di Chimica “Ugo Schiff” \& INSTM RU, Università degli Studi di Firenze, I50019 Sesto Fiorentino, Italy \\ ${ }^{\ddagger}$ European Laboratory for Nonlinear Spectroscopy, Università degli Studi di Firenze, I50019 Sesto Fiorentino, Italy \\ ${ }^{\S}$ School of Physics, AMBER and CRANN, Trinity College, Dublin 2, Ireland \\ "Dipartimento di Fisica ed Astronomia, Università degli Studi di Firenze, I50019 Sesto Fiorentino, Italy
}

Supporting Information

ABSTRACT: Here we report the investigation of the magnetization dynamics of a vanadyl complex with diethyldithiocarbamate $\left(\mathrm{Et}_{2} \mathrm{dtc}^{-}\right)$ligands, namely [VO$\left.\left(\mathrm{Et}_{2} \mathrm{dtc}\right)_{2}\right](\mathbf{1})$, in both solid-state and frozen solution. This showed an anomalous and unprecedentedly observed field dependence of the relaxation time, which was modeled with three contributions to the relaxation mechanism. The temperature dependence of the weight of the two processes dominating at low fields was found to well correlate with the low energy vibrations as determined by $\mathrm{THz}$ spectroscopy. This detailed experimental comparative study represents a fundamental step to understand the spin dynamics of potential molecular quantum bits, and enriches the guidelines to design molecule-based systems with enhanced quantum coherence.

uantum properties of matter such as entanglement, superposition and tunneling can be fruitfully employed to develop quantum computation, a novel computational paradigm based on the quantum bit, or qubit. ${ }^{1}$ A qubit, with respect to the bit, has the property to be placed in a coherent superposition state other than the two classical 0 and 1 states. Various physical systems can be regarded as viable qubits, and among them electronic spins are seen as promising candidates. ${ }^{2}$ Mainly, this is due to their easy manipulation through pulsed microwave radiations and electric fields. ${ }^{2 \mathrm{~b}, 3}$ Electronic defects on silicon, ${ }^{4}$ silicon carbide, ${ }^{5}$ or diamond ${ }^{6}$ are the best qubits candidates in the realm of purely inorganic materials. Although they satisfy some of the requirements to behave as viable qubits, they lack chemical tunability of their electronic/magnetic properties. Such a tunability can be instead achieved through chemical design of transition metal complexes. ${ }^{7}$ Indeed, coordination compounds, mostly based on $\mathrm{V}^{\mathrm{IV}}$ and $\mathrm{Cu}^{\mathrm{II}}, 8$ have been demonstrated as effective as electronic defects in inorganic materials potential qubits, as far as the lifetime of the superposition state is concerned. ${ }^{8 c}$ Furthermore, the ability of coherently manipulate this superposition state up to room temperature was also proven. ${ }^{8 \mathrm{~d}}$

The lifetime of the superposition state is denoted by $T_{2}$ or $T_{m}$, which are the spin-spin relaxation time, and the phase memory or quantum coherence time, respectively. Several efforts have been done in the last years to understand the factors affecting the absolute values and the temperature dependence of $T_{m}$. These studies provide specific design criteria to develop potential qubits with improved performances. ${ }^{8 \mathrm{e}, 9}$

Another key parameter to evaluate the performances of a spin qubit is the spin-lattice relaxation time, $T_{1}$, which defines the time required to an excited spin to relax back to its ground state. Control of $T_{1}$ is of paramount importance; if too long it limits the speed of initialization of the qubit, while if too short it can limit $T_{2}{ }^{8 \mathrm{e}}$ This means that to enhance both the absolute value of $T_{m}$ and its operational temperature range, a judicious control of $T_{1}$ is required. By introducing a unique structural difference, i.e., an oxovanadium(IV) in a square pyramidal versus a vanadium(IV) in an octahedral environment featuring the same coordinating ligand, we have recently demonstrated the effect of the vanadyl moiety in enhancing quantum coherence up to room temperature. ${ }^{8 \mathrm{e}}$ This has been related to a less effective mechanism of spin-lattice relaxation, which can be evaluated by the exponent $n$ (ca. 3 ) of the $T$ dependence of the relaxation rate, $T_{1}{ }^{-1}$, when compared to the nonoxo counterpart $(n=4)$. Indeed, the latter shows a more rapid collapse which hampers the observation of quantum coherence at room temperature, while the former reaches record $T_{m}$. Despite the relevance of the $n$ exponent and its general relation to the stiffness of the lattice, i.e., to the Debye temperature $\theta_{\mathrm{D}}{ }^{10}$ clear correlations with the structural and electronic parameters of the spin center have not been established yet.

Moved from the interest to better understand the relation between relaxation mechanisms and vibrational energies in potential molecular qubits, we have selected as a promising candidate the diethyldithiocarbamate complex $\left[\mathrm{VO}\left(\mathrm{Et}_{2} \mathrm{dtc}\right)_{2}\right]$ (1). Its magnetization dynamics, studied by alternate current (AC) susceptometry, showed an anomalous and unprecedentedly observed field dependence of the relaxation time. A detailed analysis revealed important insights on the role of low energy vibrations, experimentally detected by $\mathrm{THz}$ spectroscopy, on this behavior.

Compound 1 can be obtained by reaction of vanadyl sulfate with sodium diethyldithiocarbamate in a disareated water solution, similarly to what was already reported. ${ }^{11}$ The

Received: February 6, 2017

Published: March 6, 2017 
molecular structure of $\mathbf{1}$ shows a square pyramidal coordination geometry with the $\mathrm{V}^{\mathrm{IV}}$ ion above the basal plane $(0.75 \AA)$. The apical position is occupied by an oxo ligand that forms a double bond with the $\mathrm{V}^{\mathrm{IV}}$ ion. The $\mathrm{V}=\mathrm{O}$ bond distance is $1.591 \AA$ long and it is in the usual range for vanadyl complexes. ${ }^{8 \mathrm{~d}, \mathrm{e}, 9 \mathrm{~b}, \mathrm{c}, 12}$ On the other coordination sites the metal is chelated by two $\mathrm{Et}_{2} \mathrm{dtc}^{-}$anions (Figure 1).

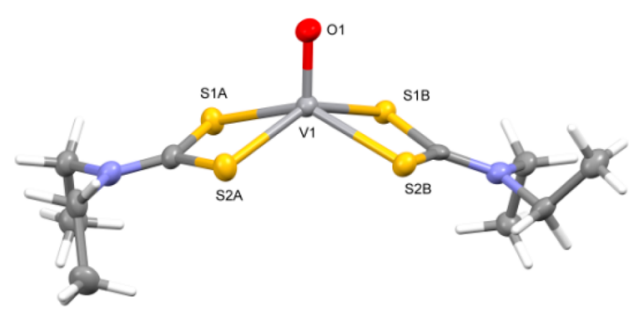

Figure 1. Molecular structure of 1 with principal atoms labeling scheme. A and B refer to two crystallographically independent ligands.

The V-S single bond lengths are in the 2.395-2.414 $\AA$ range and are slightly longer than those observed for vanadyldithiolenes complexes. ${ }^{8 \mathrm{e}, 9 \mathrm{~b}, \mathrm{c}}$ The presence of such a short $\mathrm{V}=$ $\mathrm{O}$ bond is responsible for a $d$-orbitals splitting of the metal centers in which the $d_{x y}$ orbital lies lowest in energy and well separated from the other orbitals. This quenches the orbital contribution to the ground state when compared to other coordination geometries, as the pseudo-octahedral one, and makes 1 a potential qubit with expected enhanced performances. Interestingly, the terminal methyl groups of one $\mathrm{Et}_{2} \mathrm{dtc}^{-}$ ligand are oriented, as expected, above and below the $\mathrm{S}_{2} \mathrm{CN}$ plane of the ligand, whereas in the other one they are both oriented in the same direction (Figure 1). This sterically unfavorable orientation may be driven from the presence of short-contacts involving the terminal methyl groups (Figure S1). ${ }^{11}$ Powder X-ray diffraction analysis performed on $\mathbf{1}$ allowed to ascertain structural phase homogeneity (Figure S2).

Continuous-wave EPR spectra on frozen solution were recorded to have a full description of the electronic properties of 1 (Figure S3). The spectrum shows the 8-fold hyperfine splitting due to the coupling between the $S=1 / 2$ electronic spin of $\mathrm{V}^{\mathrm{IV}}$ and the $I=7 / 2$ nuclear spin of ${ }^{51} \mathrm{~V}$ (natural abundance $99.76 \%$ ). The signal is further split by the anisotropic components of the Lande factor and the hyperfine coupling. Spectral simulations ${ }^{13}$ performed on the basis of the following spin Hamiltonian

$$
\mathcal{H}=\hat{I} \cdot \mathbf{A} \cdot \hat{S}+\mu_{B} \hat{S} \cdot \mathbf{g} \cdot \mathbf{B}
$$

allow one to simulate the spectrum of $\mathbf{1}$ with the following parameters: $g_{x}=1.985(1), g_{y}=1.989(1), g_{z}=1.969(1) ; A_{x}=$ 153(2) $\mathrm{MHz}, A_{y}=135(2) \mathrm{MHz}, A_{z}=440(2) \mathrm{MHz}$ (Figure S3).

The relaxation time $\tau$, which is representative of the spinlattice relaxation, was investigated by $\mathrm{AC}$ susceptometry as a function of the temperature at four selected static magnetic field values. When a small static magnetic field $(>40 \mathrm{mT})$ is applied, 1 shows slow relaxation of the magnetization. The frequency dependence of the imaginary component of the susceptibility $\left(\chi^{\prime \prime}\right)$ are well reproduced with the Debye model (Figures S4-S7) and the extracted values of $\tau$ as a function of $T$ are reported in Figure 2a. Slow magnetic relaxation is observed in the entire studied $T$ range $(4.0-50 \mathrm{~K})$ with quite long relaxation times. They range from ca. $5.0 \mathrm{~ms}$ at $4.0 \mathrm{~K}$ to ca. 0.03
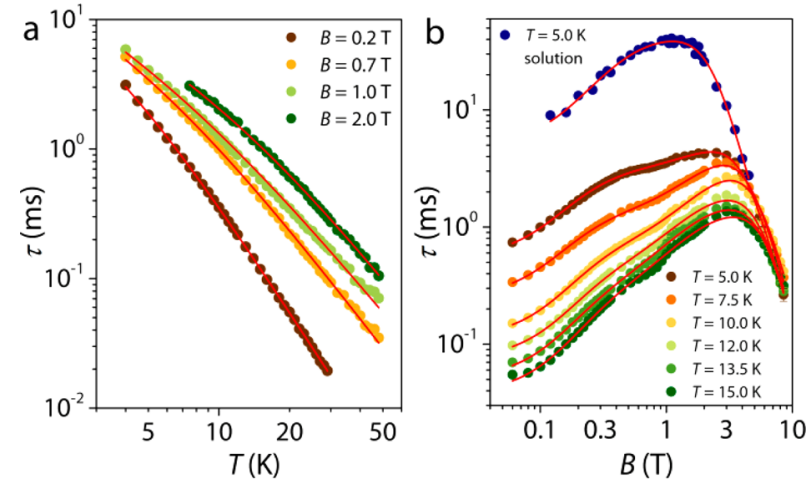

Figure 2. Temperature (a) and field (b) dependence of $\tau$ for $\mathbf{1}$ at different static magnetic fields and temperatures (see legends). Solid lines are the best-fits of the models (see text).

$\mathrm{ms}$ at $50 \mathrm{~K}$. As the strength of the magnetic field increases, longer relaxation times are observed. The $T$ dependence of $\tau$, reveals, independently from the strength of the applied field, a change in the slope at low temperature (Figure 2a), which is indicative of a change in the nature of the main relaxation mechanism involved. Thus, the $T$ dependence of the relaxation rate $\left(\tau^{-1}\right)$ has been modeled assuming two contributions to the relaxation. A direct mechanism dominating at low temperature and a Raman mechanism dominating at high temperature

$$
\tau^{-1}=a T+b T^{n}
$$

where $a$ is the coefficient of the direct mechanism, and $b$ and $n$ the coefficient and the exponent of the Raman mechanism, respectively. This model satisfactorily reproduces the $T$ dependence of $\tau$ with the best-fit parameters reported in Table S1. The fit furnishes very low values of the Raman exponent $n<3$, which are not unusual for vanadyl systems, as recently found. ${ }^{8 \mathrm{~d}, \mathrm{e}}$ Attempts to reproduce the data either assuming an Orbach process or expanding the Raman term according to the Debye model ${ }^{10}$ (Figure S8) were unsuccessful, the latter providing a field dependent $\theta_{\mathrm{D}}$.

To get deeper insights on the relaxation mechanisms involved, the dynamics was studied as a function of the static magnetic field in a wide field range $(0.0-8.5 \mathrm{~T})$ at different temperatures (Figures S9-S14). The extracted relaxation times are reported in Figure $2 \mathrm{~b}$. The field dependence of $\tau$ for 1 shows (i) very long relaxation times, and (ii) an anomalous and unprecedentedly observed behavior. The relaxation time shows a rapid increase at low fields, reaches an apparent maximum value at ca. $0.5 \mathrm{~T}$, stays almost constant up to ca. $1.0 \mathrm{~T}$, and then suddenly increases further up to ca. 3.0 T. If the field is increased further, $\tau$ quickly collapses to ca. $0.3 \mathrm{~ms}$. Previously investigated vanadyl systems have shown a more regular behavior: $\tau$ rapidly reaches a maximum value and then remains almost unchanged up to ca. $3.5-4 \mathrm{~T}^{8 \mathrm{~d}, \mathrm{e}, 12}$ The behavior at high fields $(B>3.0 \mathrm{~T})$ is related to the more efficient spin-phonon direct mechanism of relaxation $\left(\tau^{-1} \propto B^{4}\right)$. Indeed, the larger is the energy separation of the two $m_{S}$ states, the higher is the phonon density with an energy corresponding to this difference. At low fields $(B<0.3 \mathrm{~T})$, the relaxation is instead promoted by spin-spin and spin-nuclei interactions. These interactions are suppressed by increasing the field due to the lower influence of the hyperfine and spin-spin coupling, so that $\tau$ usually shows maximum values at intermediate fields.

What it is unusual here is the presence of a pronounced shoulder at ca. $0.5 \mathrm{~T}$, suggesting that more than two 
contributions to the relaxation are involved in determining the $B$ dependence of $\tau^{-1}$ for $\mathbf{1}$. To exclude that this effect is related to inhomogeneous crystallite sizes, and, consequently, to spinphonon bottleneck effects, ${ }^{12}$ the measurements were also repeated on a finely milled sample. No appreciable differences were observed in the two morphological states, and only a small discrepancy in the absolute values of $\tau$ is evidenced (Figure S15).

Prior to this work, the magnetic field dependence of the relaxation rate was reproduced with the Brons-van Vleck model, which takes into account the two above-mentioned contributions to the relaxation through the following expression $^{14}$

$$
\tau^{-1}=c B^{4}+d \frac{1+e B^{2}}{1+f B^{2}}
$$

where the first term is related to the direct mechanism, and the second term takes into account the effect of the internal magnetic field in promoting relaxation. This can be viewed as the sum of intra- and intermolecular effects, i.e., the sum of spin-nuclei hyperfine interactions and spin-spin dipolar magnetic interactions. More specifically, $d$ represents the relaxation at zero field, $f$ the ability of the external magnetic field to suppress the internal relaxation mechanisms, and $e$ the attitude of the internal magnetic moments to induce relaxation. This last may be considered proportional to the number and strength of the internal spins. This model does not reproduce the $B$ dependence of $\tau$ shown by $\mathbf{1}$ due to the anomalous behavior at intermediate fields. An analysis of the various magnetic field regions suggests that for $B>3.0 \mathrm{~T}$ the relaxation rate decay is well reproduced through a $B^{4}$ dependence for all temperatures, as expected for the direct mechanism, while at intermediate fields the dependence of the relaxation rate suggests the presence of two distinguishable contributions of the internal-field-type. Accordingly, the expression of the Brons-van Vleck model (eq 3) has been extended as follows

$$
\tau^{-1}=c B^{4}+d \frac{1+e B^{2}}{1+f B^{2}}+g \frac{1+e B^{2}}{1+h B^{2}}
$$

This model well reproduces the $B$ dependence of $\tau^{-1}$ (red lines in Figure 2b) with the best-fit parameters reported in Table S2. It must be stressed here that at any field and temperature only one relaxation time with a narrow distribution is observed. Thus, eq 4 refers to three intrinsic relaxation mechanisms of the same molecular species.

It emerges from this analysis that (i) the efficiency of the direct mechanism to promote relaxation at high fields is almost temperature independent in the investigated $T$ range, (ii) the attitude of the internal spins to promote relaxation does not significantly change and allows to fit well all the $B<3.0 \mathrm{~T}$ region, (iii) the $T$ dependences of both $d$ and $g$ parameters follow an exponential growth, thus suggesting that some welldefined energy levels are involved in the relaxation mechanism. Even if the overall $T$ dependence of $\tau^{-1}$ does not follow an Arrhenius law, the individual single processes described by the $d$ and $g$ parameters actually seem to be proportional to $\exp \left(U_{\text {eff }} / k_{\mathrm{B}} T\right)$. The plots of $\ln (d)$ and $\ln (g)$ vs $T^{-1}$ (Figure $3 \mathrm{a}, \mathrm{b})$ are in fact almost linear over a relatively wide $T$ range and the corresponding linear fit provides $U_{\text {eff }}$ values of ca. $20(2)$ $\mathrm{cm}^{-1}$ and $15(2) \mathrm{cm}^{-1}$ for $d$ and $g$ respectively, which well match with the typical energies of low energy vibration modes. ${ }^{12}$ An analogous analysis has been thus performed on a microcrystal-
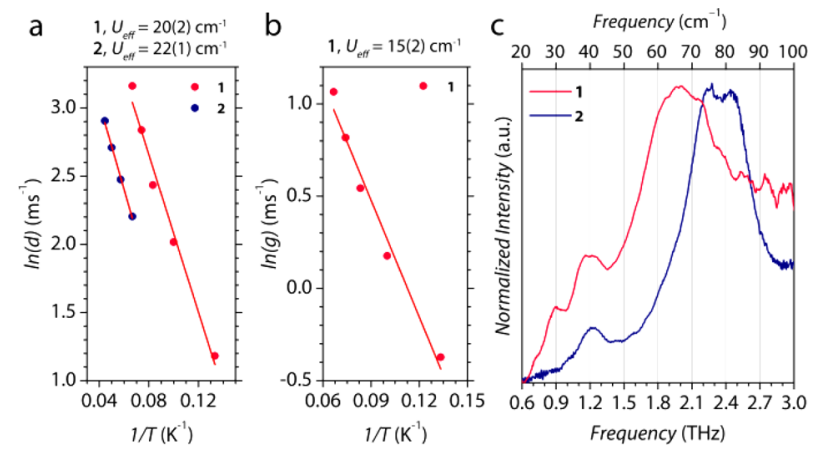

Figure 3. Arrhenius plots of $d$ (a) and $g$ (b) parameters, and vibrational spectra in the $0.6-3.0 \mathrm{THz}$ range (c) for 1 and 2 .

line sample of a previously investigated vanadyl-complex, $\left[\mathrm{VO}(\mathrm{acac})_{2}\right](\mathrm{acac}=$ acetylacetonate $)(\mathbf{2}),{ }^{12}$ which has been taken as reference. 2 shows a $B$ dependence of $\tau^{-1}$ that can be described through the simpler Brons-van Vleck model (eq 3), with only one direct and one Raman mechanism (Figure S16 and Table S3). The $T$ dependence of the $d$ parameter furnishes for 2 an $U_{\text {eff }}$ of ca. $22(1) \mathrm{cm}^{-1}$.

To validate the hypothesis that vibrational modes are involved in the relaxation, room temperature time-domain $\mathrm{THz}$ spectroscopy $(0.1-3.0 \mathrm{THz}$ range) was employed to record high signal-to-noise spectra (see Supporting Information) $)^{15}$ on 1 and 2 (Figure $3 \mathrm{c}$ ). THz spectroscopy has already been used in the field of molecular magnetism to characterize the zero field splitting of $3 d$ and $4 f$ based complexes, ${ }^{16}$ but, to the best of our knowledge, its use to correlate spin dynamics and low energy vibrations is unprecedented, although the latter are attracting increasing interest. ${ }^{17}$ The spectra (Figure 3c) reveal significant differences for the two compounds. In particular, the lowest energy vibration is observed at ca. 40 $\mathrm{cm}^{-1}$ for 2, whereas two well separated peaks at ca. 30 and 40 $\mathrm{cm}^{-1}$ are observed for 1 . Both exhibit a stronger absorption at ca. 65 and $80 \mathrm{~cm}^{-1}$, respectively. The energy values of the vibrational modes well correlates with the extracted activation energies of the relaxation mechanism in competition with the direct process. A relation of the type $U_{\text {eff }}=h \omega_{\mathrm{v}} / 2$ seems to hold. Interestingly, this dependence was recently theoretically predicted for the relaxation of $S>1 / 2$ systems characterized by easy-axis magnetic anisotropy, i.e., single molecule magnets (SMM), when excited spin levels with lower $\left|m_{S}\right|$ are much higher in energy than the low energy optical phonons. ${ }^{18}$ It thus appears that $S=1 / 2$ spin systems can be seen as a limiting case of this model.

Thus, although the field dependence of the spin dynamics of $S=1 / 2$ systems at high fields is clearly described through the direct mechanism of relaxation, at low and intermediate fields a relaxation mechanism dependent on the frequency of the low energy vibrational modes seems involved. Interestingly, the detailed analysis of the field dependence on $\mathbf{1}$ has allowed to disentangle the two contributions coming from the two low energy vibrational modes. As these modes, most likely, involve vibrations distributed on the molecular periphery and strongly admixed with lattice vibrations, a less discontinuous behavior should be observed in frozen solution where the asymmetry of the ethyl groups, induced by the intermolecular contacts, is lost. To corroborate this hypothesis, the magnetization dynamics of 1 was investigated as a function of the magnetic field at $T=5 \mathrm{~K}$ in a $\mathrm{CH}_{2} \mathrm{Cl}_{2} /$ toluene frozen solution. The relaxation times extracted with the Debye model (Figure S17) are reported in 
Figure 2b. A striking change in the $B$ dependence of $\tau$ is observed when passing from the solid-state to the solution. Whereas at high fields the relaxation time decay is that typical of the direct process, at low fields the pronounced shoulder of the crystalline phase is not observed, and the data can thus be well reproduced with eq 3 (Table S2). This suggests that the asymmetry of the ethyl groups on the ligand, most likely lost in solution, plays a key role. A detailed theoretical analysis is necessary to confirm this hypothesis, as well as to elucidate why the two processes in $\mathbf{1}$ exhibit different field dependences. We can only anticipate that distinct vibrations can affect different parameters of the spin Hamiltonian, resulting in eigenstates composition which might then be differently affected by the applied field.

In conclusion, this study represents a first attempt to correlate experimentally the spin relaxation parameters to the vibrational spectrum of molecular systems of interest as potential qubits. Low energy vibrations, whose role has been recently highlighted for anisotropic magnetic molecules, seems to govern the spin dynamics in a wide field and temperature range of these vanadyl $S=1 / 2$ systems. In contrast to SMMs, where an Arrhenius law with an effective barrier not matching the spin levels is encountered, a less informative power law temperature dependence is usually observed for molecular spin qubits. A detailed analysis of the magnetization dynamics through a bidimensional AC susceptometry investigation, scanning both field and temperature, has allowed to isolate the $T$ dependence of the single processes contributing to the magnetic relaxation, and to retrieve for the first time physical parameters that seem to correlate directly with the very low energy vibration modes. The combination with $\mathrm{THz}$ spectroscopy studies contributed to shed light on the relaxation mechanism involved. If widely employed, such a multitechnique approach will allow one to establish rational synthetic strategies to protect spin quantum coherence from vibrations.

\section{ASSOCIATED CONTENT}

\section{S Supporting Information}

The Supporting Information is available free of charge on the ACS Publications website at DOI: 10.1021/jacs.7b01266.

Additional figures and tables, and experimental section (PDF)

Crystallographic data for $\mathbf{1}$ (CIF)

\section{AUTHOR INFORMATION}

\section{Corresponding Authors}

*matteo.atzori@unifi.it

*roberta.sessoli@unifi.it

\section{ORCID}

Matteo Atzori: 0000-0003-1357-6159

Lorenzo Tesi: 0000-0003-4001-8363

Stefano Benci: 0000-0001-6492-1616

Alessandro Lunghi: 0000-0002-1948-4434

Roberto Righini: 0000-0003-0260-0248

Lorenzo Sorace: 0000-0003-4785-1331

Roberta Sessoli: 0000-0003-3783-2700

\section{Notes}

The authors declare no competing financial interest.

\section{ACKNOWLEDGMENTS}

The financial support of Italian MIUR (PRIN Project 2015 HYFSRT), Fondazione Ente Cassa di Risparmio di Firenze, and European COST (CA15128 MOLSPIN) is acknowledged.

\section{REFERENCES}

(1) Nielsen, M.; Chuang, I. Quantum Computation and Quantum Information; Cambridge University Press: Oxford, 2004.

(2) (a) Leuenberger, M. N.; Loss, D. Nature 2001, 410, 789. (b) Ardavan, A.; Rival, O.; Morton, J. J. L.; Blundell, S. J.; Tyryshkin, A. M.; Timco, G. A.; Winpenny, R. E. P. Phys. Rev. Lett. 2007, 98, 057201 .

(3) Laucht, A.; Muhonen, J. T.; Mohiyaddin, F. A.; Kalra, R.; Dehollain, J. P.; Freer, S.; Hudson, F. E.; Veldhorst, M.; Rahman, R.; Klimeck, G.; Itoh, K. M.; Jamieson, D. N.; McCallum, J. C.; Dzurak, A. S.; Morello, A. Sci. Adv. 2015, 1, e1500022.

(4) Pla, J. J.; Tan, K. Y.; Dehollain, J. P.; Lim, W. H.; Morton, J. J. L.; Jamieson, D. N.; Dzurak, A. S.; Morello, A. Nature 2012, 489, 541.

(5) Tyryshkin, A. M.; Tojo, S.; Morton, J. J. L.; Riemann, H.; Abrosimov, N. V.; Becker, P.; Pohl, H.-J.; Schenkel, T.; Thewalt, M. L. W.; Itoh, K. M.; Lyon, S. A. Nat. Mater. 2012, 11, 143.

(6) Balasubramanian, G.; Neumann, P.; Twitchen, D.; Markham, M.; Kolesov, R.; Mizuochi, N.; Isoya, J.; Achard, J.; Beck, J.; Tissler, J.; Jacques, V.; Hemmer, P. R.; Jelezko, F.; Wrachtrup, J. Nat. Mater. 2009, 8, 383.

(7) (a) Troiani, F.; Affronte, M. Chem. Soc. Rev. 2011, 40, 3119. (b) Aromi, G.; Aguila, D.; Gamez, P.; Luis, F.; Roubeau, O. Chem. Soc. Rev. 2012, 41, 537. (c) Ferrando-Soria, J.; Moreno Pineda, E.; Chiesa, A.; Fernandez, A.; Magee, S. A.; Carretta, S.; Santini, P.; VitoricaYrezabal, I. J.; Tuna, F.; Timco, G. A.; McInnes, E. J. L.; Winpenny, R. E. P. Nat. Commun. 2016, 7, 11377.

(8) (a) Warner, M.; Din, S.; Tupitsyn, I. S.; Morley, G. W.; Stoneham, A. M.; Gardener, J. A.; Wu, Z.; Fisher, A. J.; Heutz, S.; Kay, C. W. M.; Aeppli, G. Nature 2013, 503, 504. (b) Bader, K.; Dengler, D.; Lenz, S.; Endeward, B.; Jiang, S.-D.; Neugebauer, P.; van Slageren, J. Nat. Commun. 2014, 5, 5304. (c) Zadrozny, J. M.; Niklas, J.; Poluektov, O. G.; Freedman, D. E. ACS Cent. Sci. 2015, 1, 488. (d) Atzori, M.; Tesi, L.; Morra, E.; Chiesa, M.; Sorace, L.; Sessoli, R. J. J. Am. Chem. Soc. 2016, 138, 2154. (e) Atzori, M.; Morra, E.; Tesi, L.; Albino, A.; Chiesa, M.; Sorace, L.; Sessoli, R. J. Am. Chem. Soc. 2016, $138,11234$.

(9) (a) Bader, K.; Winkler, M.; van Slageren, J. Chem. Commun. 2016, 52, 3623. (b) Yu, C.-J.; Graham, M. J.; Zadrozny, J. M.; Niklas, J.; Krzyaniak, M. D.; Wasielewski, M. R.; Poluektov, O. G.; Freedman, D. E. J. J. Am. Chem. Soc. 2016, 138, 14678. (c) Graham, M. J.; Yu, C.J.; Krzyaniak, M. D.; Wasielewski, M. R.; Freedman, D. E. J. J. Am. Chem. Soc. 2017, 139, 3196.

(10) Fielding, A. J.; Fox, S.; Millhauser, G. L.; Chattopadhyay, M.; Kroneck, P. M. H.; Fritz, G.; Eaton, G. R.; Eaton, S. S. J. Magn. Reson. 2006, 179, 92.

(11) Henrick, K.; Raston, C. L.; White, A. H. J. Chem. Soc., Dalton Trans. 1976, 26, 26.

(12) Tesi, L.; Lunghi, A.; Atzori, M.; Lucaccini, E.; Sorace, L.; Totti, F.; Sessoli, R. Dalton Trans. 2016, 45, 16635.

(13) Stoll, S.; Schweiger, A. J. Magn. Reson. 2006, 178, 42.

(14) (a) Van Vleck, J. H. Phys. Rev. 1940, 57, 426. (b) De Vroomen, A. C.; Lijphart, E. E.; Prins, D. Y. H.; Marks, J.; Poulis, N. J. Physica 1972, 61, 241.

(15) Tasseva, J.; Taschin, A.; Bartolini, P.; Striova, J.; Fontana, R.; Torre, R. Analyst 2017, 142, 42.

(16) Rechkemmer, Y.; Fischer, J. E.; Marx, R.; Dörfel, M.; Neugebauer, P.; Horvath, S.; Gysler, M.; Brock-Nannestad, T.; Frey, W.; Reid, M. F.; van Slageren, J. J. Am. Chem. Soc. 2015, 137, 13114.

(17) Escalera-Moreno, L.; Suaud, N.; Gaita-Ariño, A. http://arxiv. org/abs/1512.05690v2 (accessed December 5, 2016).

(18) Lunghi, A.; Totti, F.; Sessoli, R.; Sanvito, S. Nat. Commun. 2017, 8,14620 . 